\title{
Measuring nutritional risk in hospitals
}

This article was published in the following Dove Press journal:

Clinical Epidemiology

I September 2010

Number of times this article has been viewed

\author{
Henrik H Rasmussen ${ }^{1,2}$ \\ Mette Holst ${ }^{3}$ \\ Jens Kondrup ${ }^{4}$ \\ 'Centre for Nutrition and Bowel \\ Disease, Department of Medical \\ Gastroenterology, Aarhus University \\ Hospital, Aalborg, Denmark; \\ ${ }^{2}$ Department of Clinical Epidemiology, \\ Aarhus University Hospital, Aarhus, \\ Denmark; ${ }^{3}$ Centre for Nutrition and \\ Bowel Disease, Aarhus University \\ Hospital, Aalborg, Denmark; \\ ${ }^{4}$ Department of Human Nutrition, \\ Faculty of Life Sciences, University of \\ Copenhagen, Clinical Nutrition Unit, \\ Rigshospitalet University Hospital, \\ Copenhagen, Denmark
}

Correspondence: Henrik H Rasmussen Centre for Nutrition and Bowel Disease, Department of Medical Gastroenterology, Aalborg Hospital, Aarhus University Hospital, Aalborg, Denmark $\mathrm{Tel}+45-99326229 /+45-23629615$

Fax +45-99326107

Email hhr@rn.dk

\begin{abstract}
About $20 \%-50 \%$ of patients in hospitals are undernourished. The number varies depending on the screening tool amended and clinical setting. A large number of these patients are undernourished when admitted to the hospital, and in most of these patients, undernutrition develops further during hospital stay. The nutrition course of the patient starts by nutritional screening and is linked to the prescription of a nutrition plan and monitoring. The purpose of nutritional screening is to predict the probability of a better or worse outcome due to nutritional factors and whether nutritional treatment is likely to influence this. Most screening tools address four basic questions: recent weight loss, recent food intake, current body mass index, and disease severity. Some screening tools, moreover, include other measurements for predicting the risk of malnutrition. The usefulness of screening methods recommended is based on the aspects of predictive validity, content validity, reliability, and practicability. Various tools are recommended depending on the setting, ie, in the community, in the hospital, and among elderly in institutions. The Nutrition Risk Screening (NRS) 2002 seems to be the best validated screening tool, in terms of predictive validity ie, the clinical outcome improves when patients identified to be at risk are treated. For adult patients in hospital, thus, the NRS 2002 is recommended.
\end{abstract}

Keywords: nutritional risk screening, undernutrition, clinical outcome, hospital

\section{Background}

About $20 \%-50 \%$ of all patients in hospitals are found at risk of undernutrition, depending on the definition, clinical setting, and screening tool amended. A large part of these patients are at nutritional risk when admitted to hospital, and in most of the patients, undernutrition develops negatively during hospital stay. ${ }^{1,2}$ The elderly patients and patients suffering from chronic diseases are more exposed to nutritional risk than other patients. ${ }^{3-5}$ Despite three decades of collective development of knowledge, contemporary malnutrition rates do not appear to have reduced significantly. ${ }^{5-9}$ This can be prevented if special attention is paid to nutritional care of patients. Routine identification by purposeful nutritional screening is paramount as the first stage in patient care in order to identify at-risk patients, with a view to providing nutritional support if necessary. ${ }^{10,11}$ The purpose of this article is to provide a sound basis for understanding that screening procedures are aimed at different populations and to support consideration to the continuity of the nutritional care process in clinical practice, as recommended by the European Society for clinical Nutrition and Metabolism (ESPEN). 
ESPEN guidelines for nutrition screening recommend a continuity of issues to be considered in all patients admitted to hospital (Figure 1):

1. Initially on admission, a simple nutritional screening is to be done to identify patients at actual nutritional risk.

2. Subsequently, for patients at nutritional risk, a thorough nutritional assessment is to be completed.

3. This stage leads to an individual evaluation of nutritional requirements and a plan for nutrition therapy and care.

4. Monitoring and defining targeted outcome should be structured in order to reconsider therapy and care planning. Finally, information about the results of screening, assessment, planning, and monitoring should be communicated to other health care professionals when the patient is transferred either back to the community or to another institution. ${ }^{12-14}$

In order to give priority to nutritional intervention for relevant patients, nutritional screening with regard to nutritional status and acute disease has been developed and validated in different populations. ${ }^{15-19}$ There is still no clear consensus on a definition of undernutrition or on a gold standard method of identification. Nevertheless, malnutrition has been offered numerous definitions. ${ }^{20-22}$ Simply stated in this context, malnutrition is a suboptimal nutrient status appearing as a consequence of deficiency of nutrients. However, this definition neglects the numerous causes of malnutrition. An International Guideline Committee has recently proposed an etiologybased approach that incorporates a current understanding of the inflammatory response encountered in many patients. The Committee proposed the following nomenclature for nutrition diagnosis in adults in the clinical practice setting: "starvation-related malnutrition" for chronic starvation without inflammation; "chronic disease-related malnutrition" when inflammation is chronic and of a mild to moderate degree; and "acute disease or injury-related malnutrition" when inflammation is acute and of a severe degree. ${ }^{23}$ The causes of malnutrition in patients are, thus, included in the relevant definition. As such, screening tools, which neglect to include relevant parameters, may be less efficacious in identifying malnutrition risk. Up till now, disease-related malnutrition can be treated, and nutrition-related complications, such as infections, length of hospital stay (LOS), and mortality, can be improved.

The purpose of this article is to give simple guidelines as to how undernutrition, or risk for the development of undernutrition, can be detected, by proposing a set of standards that are practicable for general use in patients and clients within present health care resources.

\section{Detection - the purpose of screening}

The purpose of nutritional screening is to predict the probability of a better or worse outcome due to nutritional factors and whether nutritional treatment is likely to influence this. Outcome from treatment may be assessed in a number of ways:

1. Improvement or at least prevention of deterioration in mental and physical function.

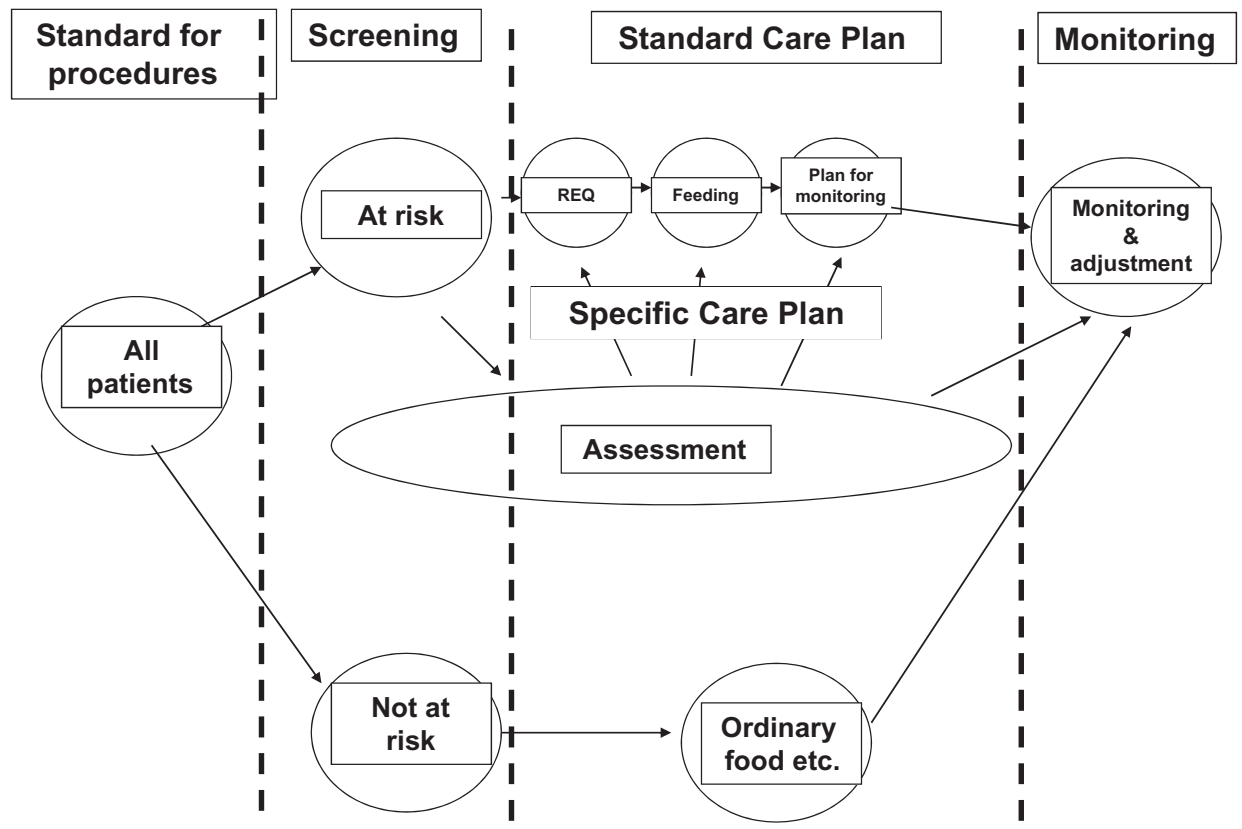

Figure I The nutritional care process including screening, planning, and monitoring according to ESPEN guidelines. ${ }^{10}$

Notes: REQ (requirements of energy and protein), feeding (ie, food, supplements, tube feeding, and parenteral nutrition), monitoring (weight and food registration). 
2. Reduced number or severity of complications of disease or its treatment.

3. Accelerated recovery from disease and shortened convalescence.

4. Reduced consumption of resources, eg, LOS and other prescriptions.

Therefore, the nutritional impairment identified by screening should be relevant to these aims and outcomes and may vary according to circumstances, eg, age or type of illness. In the community, undernutrition, with or without chronic disease, may be the primary factor determining the mental or physical function of an individual. Hence, actual undernutrition will define the outcome of nutritional treatment. In hospital, disease factors assume a greater importance with diseaseassociated undernutrition, assuming an important secondary role. Therefore, screening in the community can be focused primarily on the nutritional variables based on the results of semistarvation studies, such as those of Ancel Keys and his colleagues in $1950 .{ }^{24}$ In hospitals, other aspects of disease need to be considered in combination with purely nutritional measurements in order to determine whether nutritional support is likely to be beneficial. Randomized controlled trials (RCTs) of nutritional support in particular disease groups may, therefore, provide important evidence on which our criteria of diagnosing or defining nutritional risk are based.

\section{Evaluation - the methodology of screening}

The usefulness of screening tools can be evaluated by a number of methods. The ideal screening test has a high sensitivity (ie, it is positive in those patients who have the condition) and a high specificity (ie, it is negative in those patients who do not have the condition). A high sensitivity is important where an undetected condition has dire consequences (eg, mortality). Furthermore, it is not enough to document an association between two factors to assess the efficacy of screening tools to measure (or predict) the outcome in the future. ${ }^{25,26}$ For this purpose, it is necessary to look at the proportion of people with either a positive or negative test, which is correctly diagnosed, ie, the predictive value of the test applied. This is to avoid labeling a person with an incorrect condition (ie, "overdiagnosing"), which may cause anxiety to people who are not at risk, as well as the risk of overtreatment. ${ }^{27}$

These aspects of sensitivity, specificity, and predictive values of test results are, however, only a fraction of all aspects of the validity of a screening tool, according to Trochim and Donnelly. ${ }^{28}$ Validity focuses on whether the method is relevant to the problem under investigation. Validity is composed of several major subitems, one of which is construct validity. Construct validity describes the agreement between the diagnostic problem perceived as a concept (the construct) and what is actually done. Construct validity is composed of two main components: translation validity and criterion validity.

Translation validity deals with how the new method agrees with the construct in terms of face validity, ie, the new method seems to be right according to the experts in the field and in terms of content validity, ie, the new method includes all the elements of the problem, as seen relevant to the experts in the field, and no other elements than these. Translation validity can be obtained by including a number of experts in the development of the diagnostic method. These experts should represent the areas in which the new method will be used. For international use, the experts should also represent many countries.

Criterion validity is the comparison of the new method with other objective measures that are already accepted as having high translation validity. Predictive validity indicates that a specified event will occur in patients tested positive with the new test. For clinical use of screening in individual patients, it is not sufficient that an unfavorable event will occur as predicted. It is also required that the clinical course can be improved by treating the condition in accordance with the World Health Organization (WHO) Principles in Screening for Disease: "There should be an acceptable treatment for patients with recognizable disease" and that "Treatment at the presymptomatic, borderline stage of disease should favourably influence its course and prognosis". ${ }^{5,26}$ This means that screening for malnutrition should not only result in a likelihood of the diagnosis of malnutrition and a likelihood of adverse outcome but also in the likelihood that outcome will improve by nutritional treatment. The screening result should be useful both for diagnosis and prognosis, as well as an indication for treatment.

Other aspects of criterion validity are convergent validity, which refers to an agreement with an already established method ( $\approx$ sensitivity), and concurrent validity, which refers to not falsely identifying groups of subjects without the condition ( $\approx$ specificity). Related to these is the discriminate validity, which more specifically is a distinction from another condition in the subject, eg, the normal body mass index (BMI; height in meter ${ }^{2}$ /weight in kilograms) is not due to edema. In a clinical context, both concurrent and discriminative validity relate to specificity.

Another major subitem of validity is internal validity, which deals with the cause-effect relationships, eg, the impaired nutritional status leads to poor outcome, and the 
nutritional support improves outcome, independent of the effect of the underlying disease. Finally, external validity is the extent to which data obtained in one center are applicable to other centers, (cf the argument for multicenter, multinational studies).

The predictive validity is of major importance, ie, the individual identified to be at risk by the method is likely to obtain a health benefit from the intervention arising from the results of the screening. This can be obtained in various ways, as described for the individual screening tools below.

A screening tool must additionally have a high reliability, ie, little interobserver variation. It must also be practical, ie, those who are going to use the tool must find it rapid, simple, and intuitively purposeful. It should not contain redundant information, eg, information about vomiting or dysphagia is unnecessary when dietary intake is part of the screening. The etiology of reduced dietary intake belongs to assessment or is incorporated into the nutritional care plan. Several other aspects of evaluating screening tools are described in an analysis of 44 nutritional screening tools. ${ }^{5}$ Finally, a screening tool should be linked to specified protocols for action, eg, referral of those screened at risk to an expert for more detailed assessment and care plans.

\section{Components of nutritional screening according to ESPEN guidelines}

Screening tools are designed to detect protein and energy undernutrition and/or to predict whether undernutrition is likely to develop or worsen under the present and future conditions of the patient or client. Therefore, screening tools embody the following 4 main principles:

1. Actual condition? Height and weight allow calculation of BMI. Ranges for BMI are as follows: normal, 20-25; obesity, >30; borderline underweight, 18.5-20; and undernutrition, $<18.5$. In cases where it is not possible to obtain height and weight, eg, in some severely ill patients, a useful surrogate may be mid-arm circumference, measured at the upper-arm midway between the acromion and the olecranon. This can be related to centiles of tables for the particular population, age, and sex. ${ }^{23}$ BMI may be less useful in growing children and adolescents, and in the very elderly. Nevertheless, the BMI in general provides the best overall accepted measure of weight for height.

2. Is the condition stable? Recent weight loss is obtained from the patient's history, or even better, from previous measurements in medical records. More than 5\% involuntary weight loss for 3 months is usually regarded as significant. This may reveal undernutrition that was not discovered by measurement of height and weight or BMI, eg, weight loss in obesity. Weight loss may also predict further nutritional deterioration. ${ }^{22,29}$

3. Will the condition worsen? This question may be answered by asking whether food intake has been decreased up to the time of screening and if so by approximately how much and for how long. Confirmatory measurements can be made of the patient's food intake in hospital or by food diary. If these measurements are found to be less than the patient's requirements with normal intake, then further weight loss is likely.

4. Will the disease process accelerate nutritional deterioration? In addition to decreasing appetite, the disease process may increase nutritional requirements due to the stress metabolism associated with severe disease (eg, major surgery, sepsis, and multitrauma), causing nutritional status to worsen more rapidly or to develop rapidly from fairly normal states. ${ }^{22,23,28,30,31}$

Variables 1-3 should be included in all screening tools, whereas the fourth variable is relevant mainly in the hospital setting. In screening tools, each variable should be given a score, thereby quantifying the degree of risk and allowing a direct link to a defined course of action.

\section{Different screening tools for different settings In the hospital: Nutrition Risk Screening 2002 for adults}

Nutrition Risk Screening (NRS) 2002 was established by using a retrospective analysis of controlled trials and the nutritional criteria or characteristics and clinical outcome in these studies (Table 1). The system was developed on the assumption that the indications for nutritional support are the severity of undernutrition and the increase in nutritional requirements, resulting from the disease, ie, the severe undernutrition or severe disease by themselves or in varying combinations may indicate the need for nutritional support. This will also include patients who are not undernourished at the time but are at risk of becoming so because of disease and/ or its treatment, eg, major trauma, surgery, or chemotherapy, since both may cause impairment of food intake and increased stress metabolism. The concept of relating nutritional status to severity of disease is well recognized, as displayed for example in the decision box, which emphasizes the need for acting on possible further impairment of nutritional status during the clinical course of the disease. These concepts 
Table I Nutrition Risk Screening (NRS) 2002

\begin{tabular}{|c|c|c|}
\hline \multicolumn{2}{|c|}{ Step I: Initial screening } & \multirow[t]{2}{*}{ Yes No } \\
\hline I & Is $\mathrm{BMI}<20.5 ?$ & \\
\hline 2 & Has the patient lost weight within the last $3 \mathrm{mo}$ ? & \\
\hline 3 & Has the patient had a reduced dietary intake in the last wk? & \\
\hline 4 & Is the patient severely ill? (eg, in intensive therapy) & \\
\hline \multicolumn{3}{|c|}{ Yes: If the answer is "Yes" to any question, the screening in step 2 is performed. } \\
\hline \multicolumn{3}{|c|}{$\begin{array}{l}\text { No: If the answer is "No" to all questions, the patient is rescreened at weekly intervals. If the patient, eg, is scheduled for a major operation, } \\
\text { a preventive nutritional care plan is considered to avoid the associated risk status. }\end{array}$} \\
\hline
\end{tabular}

Step 2: Final screening
Impaired nutritional status

Absent score $0 \quad$ Normal nutritional status A

Mild score I Weight loss $>5 \%$ in $3 \mathrm{mo}$

or Severity of disease ( $\approx$ increase in requirements)

Food intake below $50 \%-75 \%$ of normal requirement in preceding wk

$\begin{array}{ll}\text { Absent score } 0 & \text { Normal nutritional requirements } \\ \text { Mild score } & \text { Hip fracture }{ }^{\mathrm{a}} \text { Chronic patients, }\end{array}$

Mild score I Hip fracture ${ }^{a}$ Chronic patients,

\section{in particular with acute} complications: cirrhosis, ${ }^{a}$ COPD ${ }^{a}$ Chronic hemodialysis, diabetes, oncology

Moderate score 2 Weight loss $>5 \%$ in 2 mo

Moderate score 2 Major abdominal surgery ${ }^{a}$ Stroke ${ }^{a}$

or Severe pneumonia, hematologic

BMI I8.5-20.5 + impaired general condition malignancy

or Food intake below $25 \%-50 \%$ of normal requirement in preceding wk

Severe score 3 Weight loss $>5 \%$ in Imo ( $>15 \%$ in $3 \mathrm{mo})$ Severe score 3

Head injury ${ }^{\mathrm{a}}$ Bone marrow or

$\mathrm{BMI}<18.5+$ impaired general condition

or

Food intake below $0 \%-25 \%$ of normal requirement in preceding wk

Score: $\quad+$

$+$

Age if $\geq 70 \mathrm{y}$ : add I to total score above transplantation ${ }^{\text {a Intensive care }}$ patients (APACHE > 10)

Score: $\quad=$ Total score:

= age-adjusted total score

Score $\geq 3$ : the patient is nutritionally at risk, and a nutritional care plan is initiated

Score $<3$ : weekly rescreening of the patient. If the patient, eg, is scheduled for a major operation, a preventive nutritional care plan is considered to avoid the associated risk status.

Notes: NRS 2002 is based on an interpretation of available randomized clinical trials. Nutritional risk is defined by the present nutritional status and risk of impairment of present status, due to increased requirements caused by stress metabolism of the clinical condition.

A nutritional care plan is indicated in all patients who are $(I)$ severely undernourished $($ score $=3)$, $(2)$ severely ill $($ score $=3)$, $(3)$ moderately undernourished + mildly ill (score $2+$ score I), or (4) mildly undernourished + moderately ill (score I + score 2).

Diagnoses shown in italics are based on the prototypes for severity of disease given below:

I. Score = I: a patient with chronic disease, admitted to hospital due to complications. The patient is weak but out of bed regularly. Protein requirement is increased, but can be covered by oral diet or supplements in most cases.

2. Score $=2$ : a patient confined to bed due to illness, eg, following major abdominal surgery. Protein requirement is substantially increased, but can be covered, although artificial feeding is required in many cases.

3. Score = 3: a patient in intensive care with assisted ventilation etc. Protein requirement is increased and cannot be covered even by artificial feeding. Protein breakdown and nitrogen loss can be significantly attenuated.

a trial directly supports the categorization of patients with that diagnosis.

Abbreviations: BMI, body mass index; COPD, chronic obstructive pulmonary disease; APACHE, acute physiology and chronic health evaluation.

are illustrated both by the study of Bastow et al ${ }^{32}$ in elderly women with fractured neck of femur, which showed that nutritional support was effective only in those patients who were particularly undernourished but not in those patients who were less undernourished, and by the study of Müller et al, ${ }^{33}$ which showed that the positive effect of perioperative nutritional support disappeared when the surgical technique was changed from a transthoracic procedure to a less-invasive stapling procedure.

This screening system, which was designed to include measures of current potential undernutrition and disease severity, was then validated against all controlled trials of nutritional support in order to evaluate whether it was capable of distinguishing those with a positive clinical outcome from those that showed no benefit from nutritional support. The analysis and the recommendations were reviewed and discussed with an ESPEN ad hoc working group under the auspices of the ESPEN Educational Committee.

The purpose of the NRS 2002 system is to detect the presence of undernutrition and the risk of developing undernutrition in the hospital setting. ${ }^{29}$ The NRS 2002 system contains the nutritional components of Malnutrition Universal Screening Tool (MUST), and in addition, a grading of severity of disease as a reflection of increased nutritional requirements. It includes 
four questions as a prescreening for departments with few at-risk patients. With the prototypes for severity of disease given, it is meant to cover all possible patient categories in a hospital. A patient with a particular diagnosis does not always belong to the same category. A patient with cirrhosis, for example, who is admitted to intensive care because of a severe infection, should be given a score of 3 , rather than 1 . It also includes old age as a risk factor, based on RCTs in elderly patients. ${ }^{19}$

\section{Evaluation}

The predictive validity of NRS 2002 has been documented by applying it to a retrospective analysis of 128 RCTs of nutritional support, which showed that RCTs with patients fulfilling the risk criteria had a higher likelihood of a positive clinical outcome from nutritional support than RCTs of patients who did not fulfill these criteria. ${ }^{29}$ In addition, it has been applied prospectively in a controlled trial with 212 hospitalized patients selected according to this screening method, which showed a reduced LOS among patients with complications in the intervention group (when adjusted for occurrence of operation and death). ${ }^{28}$ Its content validity was maximized by involving an ESPEN ad hoc working group under the auspices of the ESPEN Educational and Clinical Practice Committee in the literature-based validation. It has also been used by nurses and dieticians in 2 years of implementation study in three hospitals (local, regional, and university hospital) in Denmark, ${ }^{34}$ which indicated that staff and investigators seldom disagreed about the patient's risk status. Its reliability was validated by interobserver variation between a nurse, a dietitian, and a physician with a $\kappa=0.67$. Its practicability was shown by the finding that $99 \%$ of 750 newly admitted patients could be screened. The incidence of at-risk patients was about $20 \%{ }^{34}$

\section{In the community: MUST for adults}

The purpose of the MUST system is to detect undernutrition on the basis of knowledge about the association between impaired nutritional status and impaired function. ${ }^{8}$ It was primarily developed for use in the community.

\section{Evaluation}

The predictive validity of MUST in the community is based on the previous and recent studies of the effect of semistarvation or starvation on mental and physical function in healthy volunteers, concurrent validity with other tools, and utilization of health care resources. The new series of studies describe the impairment of function as a result of various extents of weight loss, with various rates of weight loss, from various initial nutritional statures (low or high BMI). ${ }^{2}$
It has been documented to have a high degree of reliability (low interobserver variation) with a $\kappa=0.88-1.00$. Its content validity has been assured by involving a multidisciplinary working group in its preparation. Its practicability has been documented in a number of studies in different community regions in the United Kingdom. ${ }^{8}$ The tool has recently been extended to other health care setting, including hospitals, where again it has been found to have excellent interrate reliability, concurrent validity with other tools, and predictive validity (LOS, mortality in elderly wards, and discharge destination in orthopedic patients).

\section{The elderly: Mini Nutritional Assessment}

The purpose of Mini Nutritional Assessment (MNA) is to detect the presence of undernutrition and the risk of developing undernutrition among the elderly in home-care programs, nursing homes, and hospitals. The prevalence of undernutrition among the elderly may reach significant levels (15\%-60\%) under these circumstances. ${ }^{35}$ The screening methods mentioned earlier will detect undernutrition among many elderly patients, but for the frail elderly, the MNA screening is more likely to identify the risk of developing undernutrition and undernutrition at an early stage, since it also includes physical and mental aspects that frequently affect the nutritional status of the elderly, as well as a dietary questionnaire. It is, in fact, a combination of a screening and an assessment tool, since the last part of the form is a more detailed exploration of the items in the first part of the form.

\section{Evaluation}

The predictive validity of MNA has been evaluated by demonstrating its association with adverse health outcome, ${ }^{36}$ social functioning, ${ }^{37}$ mortality, and a higher rate of visits to the general practitioner. ${ }^{15,25,27}$ In a randomized trial of elderly patients at risk according to MNA, those patients who were given oral supplements had increased body weight, but not grip strength; and in another similar (but small) randomized trial of elderly in a nursing home, the dietary intake was increased in the intervention group, but no functional or clinical outcome data were reported. ${ }^{38,39}$ However, MNA has a low efficacy with regard to predicting future malnutrition or adverse health outcomes for old people screened positive at baseline. ${ }^{40}$ Four stages in the sequence of malnutrition can be defined beginning with (1) risk factors, then progressing to (2) inadequate dietary intake relative to nutritional needs, (3) anthropometrical changes, and finally (4) measurable health outcomes. ${ }^{41}$ MNA contains items from several stages along the pathway from risk factors (eg, cognitive performance or social isolation) to adverse health 
outcomes (eg, disability or disease). This might explain the limited predictive capacity. Finally, the MNA was originally validated in relatively healthy old people. ${ }^{8}$ Hence, a number of the questions target independently living old people and not a frailer population, which have been the target population in most of the prospective studies. Also, it is wise to evaluate a screening test on old people with the same prevalence of the outcome as those for whom the test will be used in the future.

The content validity has not been reported. The reliability (interobserver variation) was estimated, with a $\kappa=0.51$. The MNA takes $<10$ minutes to complete, and its practicability has been shown by its use in a large number of studies. ${ }^{35}$

\section{Predictive validity vs meta-analyses of treatment}

The predictive validity reported here needs to be commented upon in relation to recent meta-analyses or systematic reviews. Such analyses suggest that nutritional support by the enteral or oral route improves functional capacity and clinical outcome and reduces LOS and mortality. ${ }^{16,42}$ In a meta-analysis of studies on parenteral nutrition, ${ }^{12}$ it is pointed out that there are inadequate data to assess the efficacy of parenteral nutrition in patients who are severely undernourished, who have highly catabolic disease processes, or who cannot be provided with enteral nutrition for several weeks. These are, in fact, the patients who most commonly receive supportive parenteral nutrition nowadays, and for ethical reasons, probably, there will not be any randomized trials available in the future either. Most of the studies available deal with the grey area of patients who are less undernourished or not undernourished and/or are mildly - moderately catabolic. With these studies at hand, it was difficult to identify clinical conditions where parenteral nutrition would be clinically effective. ${ }^{12}$ However, the literature analysis mentioned earlier suggests that parenteral nutrition is clinically effective in patients who rather more than just fulfill the criteria for being nutritionally at risk. ${ }^{26}$

Furthermore, nutrients known to be essential for healthy humans are also essential for patients, and therefore, the required documentation is not to confirm the essentiality of nutrients among patients, but rather to define when a certain form of nutritional support is more beneficial than leaving the patient to develop nutritional deficiencies. Therefore, metaanalyses and systematic reviews of nutritional support are too simplistic, if performed by analogy with treatment using a new drug. Finally, a nutritional care plan in most cases will involve food, oral supplements, tube feeding, and parenteral nutrition often used interchangeably in the same patient, whereas most of the randomized trials and meta-analyses have dealt with studies of single modality treatments. The predictive validity of a screening tool, therefore, cannot be directly based on meta-analyses available at present.

\section{Conclusions}

Screening should be a simple and rapid process, which can be carried out by busy admitting nursing and medical staff. It should be sensitive enough to detect all or nearly all the patients at nutritional risk. There are some advantages in registering disease severity and nutritional status, since the 2 interact. Moderate malnutrition may be more significant in the presence of severe disease. It should be capable of being scored numerically and audited and should lead to appropriate and explicit action.

Most screening tools address 4 basic questions: recent weight loss, recent food intake, current BMI, and disease severity or some other measure of predicting risk of malnutrition. In 2003, ESPEN published guidelines for nutritional screening in the community, in the hospital, and among elderly patients in institutions. The usefulness of screening methods recommended is based on aspects of predictive validity, content validity, reliability, and practicability. The NRS 2002 seems to be the best validated screening tool, in terms of predictive validity, ie, the clinical outcome improves when patients identified to be at risk are treated. Other tools have less predictive validity, ie, it has not been shown that clinical outcome improves when those identified to be at risk is treated. On the other hand, they are considered more easily applicable and have gained some acceptance in various regions in Europe.

For adult patients in hospital, it is, thus, suggested to use the NRS 2002 (Table 1). A score $\geq 3$ generates a nutrition plan in all cases. If the patient is at risk, but metabolic or functional problems prevent the standard plan from being carried out, or if there is doubt as to whether the patient is at risk, a referral should be made to an expert for more detailed assessment. These recommendations may need to be modified in the light of future experience.

\section{Disclosure}

The authors report no conflicts of interest in this work.

\section{References}

1. Keller HH, Ostbye T. Nutritional risk and time to death; predictive validity of SCREEN (Seniors in the Community Risk Evaluation for Eating and Nutrition). J Nutr Health Aging. 2003;7(4):274-279.

2. Waitzberg DL, Caiaffa WT, Correia MI. Hospital malnutrition: the Brazilian National Survey (IBRANUTRI): a study of 4000 patients. Nutrition. 2001;17(7-8):573-580.

3. Bernabeu-Wittel M, Jadad A, Moreno-Gavino L, et al. Peeking through the cracks: an assessment of the prevalence, clinical characteristics and health-related quality of life (HRQoL) of people with polypathology in a hospital setting. Arch Gerontol Geriatr. Epub 2009 Nov 12. 
4. Chima CS, Barco K, Dewitt ML, Maeda M, Teran JC, Mullen KD. Relationship of nutritional status to length of stay, hospital costs, and discharge status of patients hospitalized in the medicine service. $J \mathrm{Am}$ Diet Assoc. 1997;97(9):975-978.

5. Edington J, Boorman J, Durrant ER, et al; The Malnutrition Prevalence Group. Prevalence of malnutrition on admission to four hospitals in England. Clin Nutr. 2000;19(3):191-195.

6. Elia M, Zellipour L, Stratton RJ. To screen or not to screen for adult malnutrition? Clin Nutr. 2005;24(6):867-884.

7. Holst M, Mortensen MN, Jacobsen BA, Rasmussen HH. Efficacy of serving bedside in-between meals - an intervention study in three medical departments. e-SPEN. 2010;5(1):e30-e36.

8. Rasmussen HH, Kondrup J, Staun M, Ladefoged K, Kristensen H, Wengler A. Prevalence of patients at nutritional risk in Danish hospitals. Clin Nutr. 2004;23(5):1009-1015.

9. Reilly JJ Jr, Hull SF, Albert N, Waller A, Bringardener S. Economic impact of malnutrition: a model system for hospitalized patients. JPEN J Parenter Enteral Nutr. 1988;12(4):371-376.

10. Jie B, Jiang ZM, Nolan MT, et al. Impact of nutritional support on clinical outcome in patients at nutritional risk: a multicenter, prospective cohort study in Baltimore and Beijing teaching hospitals. Nutrition. 2009 Dec 5 [Epub ahead of print].

11. Weekes CE, Spiro A, Baldwin C, et al. A review of the evidence for the impact of improving nutritional care on nutritional and clinical outcomes and cost. J Hum Nutr Diet. 2009;22(4):324-335.

12. Kondrup J, Allison SP, Elia M, Vellas B, Plauth M. ESPEN guidelines for nutrition screening 2002. Clin Nutr. 2003;22(4):415-421.

13. Lochs H, Allison SP, Meier R, et al. Introductory to the ESPEN Guidelines on Enteral Nutrition: terminology, definitions and general topics. Clin Nutr. 2006;25(2):180-186.

14. Volkert D, Berner YN, Berry E, et al. ESPEN Guidelines on Enteral Nutrition: geriatrics. Clin Nutr. 2006;25(2):330-360.

15. Berner YN. Assessment tools for nutritional status in the elderly. Isr Med Assoc J. 2003;5(5):365-367.

16. Guigoz Y, Vellas B. The Mini Nutritional Assessment (MNA) for grading the nutritional state of elderly patients: presentation of the MNA, history and validation. Nestle Nutr Workshop Ser Clin Perform Programme. 1999;1:3-11.

17. Hubbard C. A feasibility study to determine the reliability of the Malnutrition Universal Screening Tool against anthropometric indices in determining the malnutrition risk of elderly inpatients. Proc Nutr Soc. 2008;67(OCE):E129.

18. Kersten P, McLellan L, George S, Smith JA. The Southampton Needs Assessment Questionnaire (SNAQ): a valid tool for assessing the rehabilitation needs of disabled people. Clin Rehabil. 2000;14(6):641-650.

19. Kondrup J, Rasmussen HH, Hamberg O, Stanga Z. Nutritional risk screening (NRS 2002): a new method based on an analysis of controlled clinical trials. Clin Nutr. 2003;22(3):321-336.

20. Meijers JM, Van Bokhorst-de van der Schueren MA, Schols JM, Soeters PB, Halfens RJ. Defining malnutrition: mission or mission impossible? Nutrition. Epub 2009 Nov 30.

21. Soeters PB, Reijven PL, Van Bokhorst-de van der Schueren MA, et al. A rational approach to nutritional assessment. Clin Nutr. 2008;27(5):706-716.

22. Wilson JMG, Jungner G. Principles and Practice of Screening for Disease. Geneva, Switzerland: World Health Organisation; 1968. (Public health paper No 34).

Clinical Epidemiology

\section{Publish your work in this journal}

Clinical Epidemiology is an international, peer-reviewed, open access journal focusing on disease and drug epidemiology, identification of risk factors and screening procedures to develop optimal preventative initiatives and programs. Specific topics include: diagnosis, prognosis, treatment, screening, prevention, risk factor modification, systematic

Submit your manuscript here: http://www.dovepress.com/clinical-epidemiology-journal
23. Jensen GL, Mirtallo J, Compher C, et al. Adult starvation and disease-related malnutrition: a proposal for etiology-based diagnosis in the clinical practice setting from the International Consensus Guideline Committee. Clin Nutri. 2010;29(2):151-153.

24. Keys A. Energy requirements of adults. JAMA. 1950;142(5): 333-338.

25. Jeejeebhoy KN. Nutritional assessment. Nutrition. 2000;16(7-8): 585-590.

26. Kyle UG, Pirlich M, Schuetz T, Luebke HJ, Lochs H, Pichard C. Prevalence of malnutrition in 1760 patients at hospital admission: a controlled population study of body composition. Clin Nutr. 2003;22(5):473-481.

27. Anderson CF, Moxness K, Meister J, Burritt MF. The sensitivity and specificity of nutrition-related variables in relationship to the duration of hospital stay and the rate of complications. Mayo Clin Proc. 1984;59(7):477-483.

28. Trochim W, Donnelly JP. The Research Methods Knowledge Base. 3rd ed. Mason, OH: Thomson Learning - Atomic Dog; 2007.

29. Whitby LG. Screening for disease: definitions and criteria. Lancet. 1974;2(7884):819-822.

30. Stratton RJ, Elia M. Deprivation linked to malnutrition risk and mortality in hospital. Br J Nutr. 2006;96(5):870-876.

31. Stratton RJ, Elia M. Who benefits from nutritional support: what is the evidence? Eur J Gastroenterol Hepatol. 2007;19(5):353-358.

32. Bastow MD, Rawlings J, Allison SP. Undernutrition, hypothermia, and injury in elderly women with fractured femur: an injury response to altered metabolism? Lancet. 1983;1(8317):143-146.

33. Muller JM, Thul P, Ablassmaier B. [Perioperative nutritional therapy and its relevance for postoperative outcome]. Chirurg. 1997;68(6):574-582.

34. Rasmussen HH, Kondrup J, Staun M, et al. A method for implementation of nutritional therapy in hospitals. Clin Nutr. 2006;25(3):515-523.

35. Cederholm T, Jagren C, Hellstrom K. Outcome of protein-energy malnutrition in elderly medical patients. Am J Med. 1995;98(1): $67-74$.

36. Ek AC, Unosson M, Larsson J, Ganowiak W, Bjurulf P. Interrater variability and validity in subjective nutritional assessment of elderly patients. Scand J Caring Sci. 1996;10(3):163-168.

37. Ruxton CH, Gordon J, Kirkwood L, McMillan B, Ryan E. Risk of malnutrition in a sample of acute and long-stay NHS Fife in-patients: an audit. J Hum Nutr Diet. 2008;21(1):81-90.

38. Covinsky KE, Covinsky MH, Palmer RM, Sehgal AR. Serum albumin concentration and clinical assessments of nutritional status in hospitalized older people: different sides of different coins? J Am Geriatr Soc. 2002;50(4):631-637.

39. de Luis DA, Izaola O, Cuellar L, et al. Nutritional assessment: predictive variables at hospital admission related with length of stay. Ann Nutr Metab. 2006;50(4):394-398.

40. Beck AM, Holst M, Rasmussen HH. Efficacy of the mini nutritional assessment to predict the risk of developing malnutrition or adverse health outcomes for old people. e-SPEN. 2008;3(3):e102-e107.

41. Sorensen J, Kondrup J, Prokopowicz J, et al. EuroOOPS: an international, multicentre study to implement nutritional risk screening and evaluate clinical outcome. Clin Nutr. 2008;27(3):340-349.

42. Karsegard VL, Ferlay O, Maisonneuve N, et al. [Simplified malnutrition screening tool: Malnutrition Universal Screening Tool (MUST)]. Rev Med Suisse Romande. 2004;124(10):601-605.

\section{Dovepress}

reviews, risk \& safety of medical interventions, epidemiology \& biostatical methods, evaluation of guidelines, translational medicine, health policies \& economic evaluations. The manuscript management system is completely online and includes a very quick and fair peer-review system, which is all easy to use. 